\title{
Do nurses have fair knowledge about breast cancer? A cross sectional study in king Abdul-Aziz university hospital
}

\begin{abstract}
Background: Spreading the knowledge about breast cancer (BC) is an important issue, and nurses can help in this role. Surprisingly, there is no previous study in Saudi Arabia that test nurses' knowledge on BC. This study is the first to evaluate the nurses' knowledge about BC at King Abdul-Aziz University Hospital (KAUH).

Material and method: This is a cross-sectional study conducted at KAUH in Jeddah, Saudi Arabia. The population of interest was female nurses working in KAUH from August 2015to September 2015 in the department of pediatrics, obstetrics/gynecology, surgery, and medicine. All such nurses were invited to participate via e-mail. The inclusion criteria were female nurses with at least a nursing diploma. Exclusion criteria included nurses with past or current history of $\mathrm{BC}$, student nurses, nursing educators, and nurses involved in the questionnaire pre-test. The data was collected from the questionnaire and developed to achieve the objectives of the study. The questionnaire included questions about the demographics and 20 questions about $\mathrm{BC}$ in general. One score was given to each correct answer from these 20 questions. The subjects were classified according to their final score into poor (scored seven and less), fair (scored eight to 14), and good (scored 15 and more). A Pearson Correlation was used to study the relationship between the age, nursing experience, and total score. An independent sample $t$-test was used for the family history of breast cancer.
\end{abstract}

Results: We enrolled 316 nurses; the response rate was $81 \%$. Nine participants $(2.8 \%)$ were poor in knowledge about breast cancer, 273 participants (86.4\%) were fair, and 34 participants $(10.8 \%)$ were in the good category. Age, nursing experience, and family history were not statistically significant factors.

Conclusion: Nurse involved in this study have a generally fair understanding about BC. Age, nursing experience, and family history of $\mathrm{BC}$ do not influence their knowledge.

Keywords: nurses, knowledge, breast cancer, Saudi Arabi
Volume 9 Issue 5 - 2018

\author{
Mohammed A Almarghoub \\ Department of Surgery, King Faisal Specialist Hospital \& \\ Research Centre, Saudi Arabia
}

\begin{abstract}
Correspondence: Mohammed A Almarghoub, Plastic and Reconstructive Surgery Section, Department of Surgery, King Faisal Specialist Hospital \& Research Centre, P.O. Box 3354, Riyadh II I I , Saudi Arabia,

Tel 00966 || 44 27752, Mobile 0096653005 I226,

Email Almarghoubm@gmail.com
\end{abstract}

Received: September 26, 2018 | Published: September 26, 2018

\section{Background}

Worldwide, breast cancer (BC) is the most common cancer and the second leading cause of cancer death in women. ${ }^{1}$ Breast cancer is the most common cancer among women in Saudi Arabia. The Saudi Cancer Registry (SCR) reported 8,054 cases in $2006 .{ }^{2}$ Saudi lifestyle changes have increased cancer incidence. ${ }^{3}$ By 2025, the BC incidence will likely increase by around $350 \%$ and the mortality by around $160 \%{ }^{4}$

$\mathrm{BC}$ is a major health issue. Various studies have concluded that screening and early detection can lead to a better chance of survival. ${ }^{5}$ Early detection improves survival in one third of patients. ${ }^{6}$ Secondary prevention via screening programs and awareness campaigns plays a crucial role in decreasing cancer mortalities. ${ }^{7}$ It is especially important for health worker to have good knowledge about their risk factors and screening methods.

Spreading knowledge about $\mathrm{BC}$ in our community is a major issue-this can be done via various channels. Previous studies showed that the preferred source of such information for patients was female health care providers. ${ }^{8}$ In addition to clinicians, female nurses can play a unique role in spreading knowledge to patients and educating them about $\mathrm{BC}$ screening services and practices. ${ }^{8}$ They can offer information for their families and friends. ${ }^{7}$ Hence, it is important to assess their knowledge.

Various studies have been done in Saudi Arabia to test the BC knowledge in medical students, nursing students, and healthcare providers. However, there is surprisingly little information in the literature about $\mathrm{BC}$ knowledge in nurses. This study evaluated the BC knowledge among nurses at King Abdul-Aziz University Hospital (KAUH).

\section{Materials and methods}

This is a cross-sectional study conducted at KAUH in Jeddah, Saudi Arabia. The population of interest was female nurses working in KAUH from August 2015 to September 2015 in pediatrics, obstetrics/ gynecology, surgery, and medicine departments. All relevant nurses were invited to participate via e-mail. The inclusion criteria were female nurses with at least a nursing diploma. Exclusion criteria included nurses with past or current history of BC, student nurses, nursing educators, and nurses involved in the questionnaire pre-test. 
Ethical approval was obtained from the Ethical Committee of Faculty of Medicine in King Abdulaziz University. Written informed consent was obtained from all subjects; they were assured of the confidentiality of their responses.

The data was collected via a self-administered, pre-tested questionnaire written in English language. There is no standard questionnaire to assess the objectives of this study; thus, the questionnaire was developed from earlier studies found in the literature and modified accordingly. To improve the clarity, the questionnaire was pre-tested on three consultants and five nurses working in the hospital. The nurses who performed the pre-test were not included in the final study. The questionnaires were distributed in person to the nurses in the hospital, and an immediate response was requested.

The questionnaire contained three sections. The first section included demographic questions: age, nursing experience in years, current or past history of $\mathrm{BC}$, and family history of $\mathrm{BC}$. The second section involved ten questions about the risk factors of $\mathrm{BC}$ according to the current literature. Three answer were available to each question: "yes", "no", and "not sure". To calculate the score in this section, correct answers were given one point; wrong or not sure responses were scored as zero. The scores for each question were then summed to calculate the total score in this section.

The last section involved ten questions about general knowledge on BC. "True", "false", and "not sure" were the available answers in this section. To calculate the score in this section, correct answers were given one point; wrong or not sure responses were scored as zero. The scores for each question were then summed to calculate the total score in this section.

Finally, the participant's score in the second and third sections were added to calculate the total score. The maximum possible score was 20. The minimum possible score was zero. The subjects were then classified according totheirtotal score into three categories: poor (scored 7 and less), fair (scored 8 to 14), and good (scored 15 and more). Finally, the statistical significance between different variables (age, nursing experience in years, family history of BC) in this study and the total score were studied. SPSS version 17 was used for data analysis. The mean or the median was used according to the data distribution curve. A significant $\mathrm{p}$-value was defined as $<0.05$.

\section{Results}

We enrolled 316 subjects; the response rate was $81 \%$ and all were women. Of the 316 subjects, $81.6 \%$ were married, $15.5 \%$ were single, and $2.8 \%$ were divorced or widowed. Only $9.8 \%$ of them had a family history of BC. Measures of central tendency were computed to summarize the data for the age (in years) and the nursing experience (in years). Measures of dispersion were computed to understand the score variability for these variables. The manage $(\mathrm{M})$ was 35.27 with a standard deviation (SD) of 7.37years of nursing experience are $\mathrm{M}=10.92$ with a $\mathrm{SD}=6$. Table 1 offers more details on these demographics.

Knowledge about $\mathrm{BC}$ risk factors (second section of the questionnaire) was evaluated by ten questions (Table 2). Only two participants scored the maximum 10; two subjects also got the minimum score. The mean score of the subjects was $4.8(\mathrm{SD}=1.5)$.

Ten questions were also used to test the subject's general knowledge about BC in the last section (Table 2). Six subjects scored the maximum 10 . No one scored zero, and only one nurse scored one. The mean score was $6.6(\mathrm{SD}=1.4)$.
Table I Demographics of the respondent

\begin{tabular}{|c|c|}
\hline Total number of the respondents & 316 \\
\hline Age(years) & $\mathrm{n}(\%)$ \\
\hline Less than 25 & $10(3.2)$ \\
\hline 26 To 35 & $166(52.5)$ \\
\hline \multirow[t]{2}{*}{ More than 36} & $140(44.3)$ \\
\hline & $M=35.27, S D=7.37$ \\
\hline Marital Status & $\mathrm{n}(\%)$ \\
\hline Single & $49(15.5)$ \\
\hline Married & $258(81.6)$ \\
\hline Divorced/Widow & $9(2.8)$ \\
\hline No. of Years Working in Nursing & $\mathrm{n}(\%)$ \\
\hline Less Than 5 & $55(17.4)$ \\
\hline From 6 To 10 & $127(40.2)$ \\
\hline From 11 To 15 & $75(23.7)$ \\
\hline \multirow[t]{2}{*}{ More Than 16} & $59(18.7)$ \\
\hline & $M=10.92, S D=6$ \\
\hline Family History of Breast Cancer & $\mathrm{n}(\%)$ \\
\hline Positive History & $3 I(9.8)$ \\
\hline No History & $285(90.2)$ \\
\hline
\end{tabular}

The final score (maximum of 20) was calculated by adding the score in the previous two sections for each participant. Two subjects scored 17, which was the highest score. The lowest score wassix as achieved bythree subjects. The mean score was $11.4(\mathrm{SD}=2.2)$.

The subjects were classified according to their final score into poor (scored seven and less), fair (scored eight to 14), and good (scored 15 and more).Nine participants $(2.8 \%)$ were in the poor category, 273 participants $(86.4 \%)$ were fair, and 34 participants $(10.8 \%)$ were good (Table 2)

A Pearson product-moment correlation coefficient was computed to assess the relationship between the age and the total score. There was no correlation between the two variables $(r=0.104, n=316$, $\mathrm{p}=0.065$ ). There is no correlation between age and knowledge about $\mathrm{BC}$ in this study.

The Pearson product-moment correlation coefficient was used again to assess the relationship between the nursing experience (in years) and the total score. There was no correlation between the two variables $(\mathrm{r}=0.079, \mathrm{n}=316, \mathrm{p}=0.163)$. This suggests no correlation between nursing experience and knowledge about $\mathrm{BC}$ in this study.

Preliminary assumption testing indicated that the total scores of both nurses with a history of $\mathrm{BC}$ in their family $(\mathrm{M}=11.74, \mathrm{SD}=3.09)$ and nurses without a history of $\mathrm{BC}$ in their family $(\mathrm{M}=11.43, \mathrm{SD}=2.08)$ were normally distributed. However, there was substantially more variance in the group with a positive history. Consequently, Welch's t-test was used to compare the average score between the two groups. The Levene's Test was not significant $(\mathrm{p}=0.58)$ nor was the $\mathrm{t}$-test $\mathrm{t}$ $(314)=1.47, p=0.143$, two tailed, $(95 \%$ Confidence Interval: -0.05 to 0.35 ). We conclude that there is no correlation between family history of BC and nurses' knowledge in this study. 
Table 2 Respondents' knowledge ofbreast cancer

Knowledge items

[Correct response]

\section{First Section:}

I- A positive family history of breast cancer is a BC risk factor. [True]

2- Frequent sunlight exposure increases the rate of breast cancer. [False]

$211(66.8)$

3- Smoking is related to breast cancer.

[True]

4- Using contraceptive pills increase the breast cancer.

[True]

5-The later the age of the pregnancy, the less chance of breast cancer.[False]

6- Increasing the number of pregnancies protects from cancer.[True]

7- Breast feeding protects from breast cancer.

[True]

8- Obesity showed no relation to breast cancer.

[False]

9- Young age of first menstruation is a well-recognized risk factor.[True]

I0- Late age of menopause decreases the cancer occurrence.[False]

$57(18)$

The mean score in this section $=4.8$, $S D=\mid .5$

$305(96.5)$

II- Breast cancer is the most common cancer in females.

12- Detection of breast cancer does not improve survival.

14- Hard blow to the breast may cause breast cancer in later in life.[False]

[False]

16- Breast pain is the most common presentation of breast cancer.[False]

220(69.6)

17-Women under 40 do not need clinical breast exam at all.[False]

18- Every woman older than 40 years should have a yearly clinical breast examination by a physician.[True]

19- Mammography is the best modality for diagnosis in patient under age 40.[False]

20- Mammography is recommended yearly for individualsover age 50.[True]

The mean score in this section $=6.6$, $\mathrm{SD}=1.4$

\section{Nurses Categories According to the Total Score:}

i. Poor(scored seven and less)

ii. Fair(scored eight to 14)

$273(86.4 \%)$

iii. Good (scored 15 and more)

The mean of the total score $=\mid 1.4, S D$ 2.2 


\section{Discussion}

$\mathrm{BC}$ is the most common cancer in women worldwide and the second leading cause of cancer deaths in women. ${ }^{1}$ There are various ways to decrease the burden of this disease. Early detection is particularly easy but requires an informed community. Health practitioners play a vital role because they educate their patients. Nurses are also powerful in spreading medical knowledge about common diseases. One study showed that they are preferred to doctors for information. ${ }^{8}$ Thus, it is important to teach and assess nurses. This study was conducted to get an idea about nurses understanding of this disease burden from various aspects. To the best of our knowledge, this is the first study to evaluate nurses in Saudi Arabia.

The nurses in our study have fair knowledge: $97.2 \%$ of them scored more than seven points. However, they have some weakness in recognizing different risk factors. We also studied the factors that influence their knowledge. Age, nursing experience in years, and family history of $\mathrm{BC}$ were among the data collected. We studied the effect of these factors on their knowledge. Their performance was not significantly affected by these factors.

Our data is similar to the literature. In one study in Karachi, nurses' $\mathrm{BC}$ knowledge was evaluated via ten questions. This cohort had fair knowledge with nearly $75 \%$ of them scoring more than eight out of $15 .{ }^{8}$ Indeed, our cohort has more knowledge than the medical students in another study. Another study compared knowledge between nurses and teachers and found that nurses have better knowledge. ${ }^{9}$ We found no studies detailing why there is different performance between medical staff.

This work studied the relation between knowledge and demographic variables. Age does not significantly affect knowledge. In comparison, age was found to be significant in another study. However, this study was only conducted on nursing students. ${ }^{10}$ Nursing experience (in years) was not significant in this study in contrast to prior work. ${ }^{7} \mathrm{We}$ found that a family history of $\mathrm{BC}$ is not significant similar to the work among nursing students. ${ }^{10}$

This is a cross sectional study performed by distributing questionnaires to the population of interest. To the best of our knowledge, there are no standard questionnaires that assess BC knowledge. This work modified questionnaires from the literature; thus, there is the potential for bias in selecting difficult or easy questions. Questionnaires might be completed without careful understanding of the questions because of the busy nurses' time constraints. Our population of interest was not as large as earlier studies. This might affect some of the results and relationships.
We conclude that the nurses involved in this study have a fair understanding of BC. Age, nursing experience, and a family history of $\mathrm{BC}$ do not influence this knowledge. We recommend repeating this study with a larger sample size and including multiple centers.

\section{Acknowledgements}

Special thanks to all the medical interns who distributed the questionnaire.

\section{Conflict of interest}

The author declares no conflict of interest.

\section{References}

1. Akhigbe AO, Omuemu VO. Knowledge, attitudes and practice of breast cancer screening among female health workers in a Nigerian urban city. BMC Cancer. 2009;9(1):203.

2. AlAmoudi SM, Eldeek BS, Ayoub NN, et al. Knowledge about breast cancer among male medical students, Jeddah, 2011. Life Sci J. 2012;9(3):166-170.

3. Lee E-O, Ahn S-H, You C, et al. Determining the main risk factors and high-risk groups of breast cancer using a predictive model for breast cancer risk assessment in South Korea. Cancer Nurs. 2004; 27(5):400406

4. Ibrahim E, Zeeneldin A, Bin Sadiq B, et al. The present and the future of breast cancer burden in the Kingdom of Saudi Arabia. Med Oncol. 2008;25(4):387-393.

5. Ghanem S, Glaoui M, Elkhoyaali S, et al. Knowledge of risk factors, beliefs and practices of female healthcare professionals towards breast cancer, Morocco. Pan Afr Med J. 2011;10:21.

6. Ravichandran K, Mohamed G, Al-Hamdan NA. Public knowledge on cancer and its determinants among Saudis in the Riyadh region of Saudi Arabia. Asian Pac J Cancer Prev. 2010;11(5):1175-1180.

7. Seah M, Tan S. Am I breast cancer smart? Assessing breast cancer knowledge among healthcare professionals. Singapore Med J. 2007;48(2):158-162.

8. Ahmed F, Mahmud S, Hatcher J, et al. Breast cancer risk factor knowledge among nurses in teaching hospitals of Karachi, Pakistan: a cross-sectional study. BMC Nurs. 2006;5(1):6.

9. Demirkiran F, Akdolun Balkaya N, Memis S, et al. How do nurses and teachers perform breast self-examination: Are they reliable sources of information? BMC Public Health. 2007;7:96.

10. Alsaif A. Breast self-examination among Saudi female nursing students in Saudi Arabia. Saudi Med J. 2004;25(11):1574-1578. 